This is the final peer-reviewed accepted manuscript of:

Latorre, V., Zingali, L., Bragalli, C., Domeneghetti, A., Brath, A., "Smart Water Management in Agriculture: a Proposal for an Optimal Scheduling Formulation of a Gravity Water Distribution System", 2020 - IEEE International Workshop on Metrology for Agriculture and Forestry (MetroAgriFor), Trento, 2020, pp. 257-261, doi: 10.1109/MetroAgriFor50201.2020.9277589

The final published version is available online at:

https://doi.org/10.1109/MetroAgriFor50201.2020.9277589

Rights / License:

The terms and conditions for the reuse of this version of the manuscript are specified in the publishing policy. For all terms of use and more information see the publisher's website. 


\section{Smart Water Management in Agriculture: a Proposal for an Optimal Scheduling Formulation of a Gravity Water Distribution System}

\author{
V. Latorre \\ Department of Civil, Chemical, \\ Environmental and Materials \\ Engineering \\ University of Bologna \\ Bologna, Italy \\ vittorio.latorre@unibo.it
}

\author{
A. Domeneghetti \\ Department of Civil, Chemical, \\ Environmental and Materials \\ Engineering \\ University of Bologna \\ Bologna, Italy \\ alessio.domeneghetti@unibo.it
}

\author{
L. C. Zingali \\ Department of Civil, Chemical, \\ Environmental and Materials \\ Engineering \\ University of Bologna \\ Bologna, Italy \\ lorenzo.zingali2@unibo.it
}

\author{
A. Brath \\ Department of Civil, Chemical, \\ Environmental and Materials \\ Engineering \\ University of Bologna \\ Bologna, Italy \\ armando.brath@unibo.it
}

\author{
C. Bragalli \\ Department of Civil, Chemical, \\ Environmental and Materials \\ Engineering \\ University of Bologna \\ Bologna, Italy \\ cristiana.bragalli@unibo.it
}

\begin{abstract}
Agriculture represents one of the most water demanding sectors and its role is central on defining water saving policies. In this work, we propose an improved approach to the irrigation scheduling problem, reducing water wastage while satisfying farmers' demands and crops' water needs.

For water distribution system managed with on-demand distribution approach, the efficiency of irrigation relies on the ability of the network manager (i.e., gatekeeper) to guarantee a proper service, consisting in: the irrigation scheduling, the definition of the volume of water passing through the channels at a given time, and the operations on gates and sluices to make the water reach the farms. Consequently, the irrigation scheduling inefficiencies might be limited by: i) reducing the water wastage, ii) minimizing the gatekeeper work and iii) maximizing the satisfaction of the farmers' requirements.
\end{abstract}

We propose an improved mixed-integer linear optimization formulation that adds the possibility to store water in the channels and takes seepage into account. This new formulation is able to better represent the physical behavior of the water flow in the channels network, also avoiding the presence of flooding. The proposed optimization solution is embedded within a wider monitoring framework with the intent to fully exploit the availability of a complex network of models, repositories and sensors installed in the field.

The resulting problem is solved by one of the most used optimization solvers (IBM ILOG Cplex) and tested on a synthetic benchmark. Furthermore, we validate the results on a digital copy of the network that performs a hydraulic simulation of the irrigation system. The scheduling is accepted if the water introduced in the system can satisfy farmers' requests with the considered timing and does not produce flooding.

Keywords-Water Scheduling, Optimization, Simulation

\section{INTRODUCTION}

The achievement of an optimum management solution for gravity irrigation systems that satisfy crops' water needs and farmers' demands is a fundamental goal in the context of water savage [1]. This highlights how irrigation systems improvements are strategic measures on the way to sustainable food security [2]. Based on this evidence, the research activity focuses on the development of algorithms that seek the best solution for canal management, trying to mediate between the possible objectives to be achieved $[3,4]$. The hydraulic behaviors of irrigation canals show that these systems are complex, with a dynamicity characterized by important time lags, strong nonlinearity and numerous interactions between different consecutive sub-systems [5]. One of the most complete works on irrigation scheduling using operation research techniques is the one proposed in [6], which implemented a MILP (Mixed Integer Linear Programming) approach. The irrigation requests are denoted by three parameters: irrigation start time, duration of the irrigation process and amount of water required. The final scheduling not only keeps track of the beginning of an irrigation and its duration, but also of the quantity of water flowing in the channels and the operation that the gatekeeper must perform. In fact, the water is diverted to the farms through the gates that are often maneuvered manually, thus the number of operations must be also minimized.

In this work, we propose an improved linear mixed integer formulation of the [6] approach. The formulation is solved with Cplex and validated with the use of a hydraulic simulator. The methodology ensures a more realistic representation of the water delivering process that takes into consideration the water lost due to infiltration and make the channels store water during the irrigation period without undistributed flow. In addition, the proposed solution relaxes integer constraints for the majority of the integer variables of the initial formulation

\section{WATER ALLOCATION AND PROBLEM FORMULATION}

Water distribution systems that allocates water to the farmers are typically made of open canals and free-flow pipes that need to be managed by operators (Water Distribution Gatekeeper-WDG- or gatekeeper from now on). In particular, the irrigation networks have been designed following a hierarchical structure, in which major canals collect water from several sources and bring that water to the farmers by 
means of a series of gradually smaller canals and pipes. The delivering of water to remote farmers forces the filling of canals and pipes, which could be characterized by water losses, for many kilometers, which entails the use of a relevant water volume that is not always recoverable for irrigation purposes. In this scenario, adopting an optimal irrigation scheduling is essential to ensure a proper use of manpower and water resources.

Typically, water allocations to farmers is scheduled adopting an arrangement-based approach. This means that water demands (off-takes) placed by users (i.e., farmers) are collected by the irrigation manager over a scheduling lasting from 1-2 days up to a week, according to the water irrigation needs. This scheduling is the result of a trade-off among different interests, as well as possible general management rules. Once identified, the scheduling must then be shared with the farmers in advance (e.g. 1 or 2 days) in order to check their availability. The identification of a proper scheduling is fundamental to reduce service costs and water saving. All these considerations need to respect the maximum hydraulic capacity of the network, irrigation equipment constraints of the farmers, water travel time across the network to reach the farms, or other case specific conditions. Depending on the level of automation of the irrigation district, all or a portion of these activities are made manually by the gatekeeper, who also has additional duties. Thus, the optimal scheduling must also consider the time required for each WDG operation, including the travel time from one gate to the following one, as well as the working time during a day.

Starting from these general considerations, we divide the time horizon into $\mathrm{N}$ time intervals, and for each of them the optimization problem returns the quantity of water flowing into each canal, the operation on the gates and the on-going irrigations. In the following, the duration of the time intervals is indicated with $\delta t$.

The objective function is composed by several terms that take into account not only the satisfaction of the users, but also the needs of the water distributor, like minimizing the travelling times of the gatekeeper and the water loses. The final optimization formulation is also composed of equality and inequality constraints that model the aforementioned characteristics of the scheduling task, the physical set up of the network and the management rules. We consider the mixed-integer linear optimization problem:

$$
\begin{array}{ll}
\min _{x} & f^{T} x \\
\text { s.t. } & A x \leq b \\
& \text { Aeqx }=b e q \\
& l b \leq x \leq u b
\end{array}
$$

where $x$ is the vector containing all the variables of the problem on the time horizon, $f$ are the coefficients of the objective function, $A$ is the matrix of the linear inequality constraints, $b$ is the vector of the known terms for the linear inequality constraints, $A e q$ and $b e q$ are the matrix and the known terms for the linear equality constraints, respectively, while $l b$ and $u b$ are respectively the lower and upper bounds of the variables.

\section{TeST AND VALIDATION ON A SYNTETICH NETWORK}

In order to evaluate the methodological approach and validate the reliability of the results, the optimization model has been tested in a simplified irrigation district, which behaviors can be reproduced and understood with high degree of confidence. The synthetic case study is composed of four canals, the first two being open canals and the second two being final pipes (see Figure 1). The entrance of the water in the system is regulated by a weir gate, while an adjustable weir gate allows the water flowing into canal 2. Canals 3 and 4 are powered by canal 2 and their flow is regulated by different orifices. White diamonds in Figure 1 represent the location of water off-takes due to potential irrigations.

The benchmark we consider for our application has 10 offtakes (grey boxes in Figure 1) to be supplied in a $12 \mathrm{~h}$ period manually operating the four gates by a gatekeeper with a time interval $\delta t=1 \mathrm{~h}$. The benchmark we consider has 10 offtakes to be supplied in a $12 \mathrm{~h}$ period. We suppose that the irrigation period starts at 6:00 in the morning and stops at 18:00. The four gates upstream the channels are operated manually by a gatekeeper, whose resting period is settled between the 12:00 and the 13:00.

The simplified characteristics of the channels, as seen by the optimiser, are reported in Table 1 . The maximum value for $\tau$ (60 min) is smaller than 60 minutes and $\delta t$ is set to $60 \mathrm{~min}$. The seepage ratio $\gamma$ is set to 0.2 for all the channels resulting in a non-trivial quantity of water lost during the irrigation.

\begin{tabular}{|r|r|r|r|r|}
\hline Channel & $\tau(\mathrm{min})$ & $\mathrm{C}\left(\mathrm{m}^{3}\right)$ & \multicolumn{1}{l|}{$\gamma$} & $\rho$ \\
\hline 1 & 50 & 1152 & 0.2 & 1 \\
\hline 2 & 30 & 752.4 & 0.2 & 0.8 \\
\hline 3 & 7 & 824.4 & 0.2 & 1 \\
\hline 4 & 7 & 824.4 & 0.2 & 1 \\
\hline
\end{tabular}

Tab. 1: The specific channel characteristics for the optimiser: water travelling time $\boldsymbol{\tau}$, maximum volume that can pass through the gate per time interval (C), seepage rate $\boldsymbol{\gamma}$ and minimum quantity of water delivered within a given channel ( $\rho$; expressed as rate of $\mathrm{c}$ ).

\begin{tabular}{|l|r|r|r|r|}
\hline$\psi_{i j}$ & G1 & G2 & \multicolumn{1}{l|}{ G3 } & \multicolumn{1}{l|}{ G4 } \\
\hline G1 & 0.42 & 0.75 & 1 & 1 \\
\hline G2 & 0.75 & 0.33 & 0.5 & 0.5 \\
\hline G3 & 1 & 0.5 & 0.25 & 0.33 \\
\hline G4 & 1 & 0.5 & 0.33 & 0.25 \\
\hline
\end{tabular}

Tab. 2: The gatekeeper travelling times between the different gates upstream the channels.

Gatekeeper's travel times from gate to gate (which are comprehensive of the time required for the gate operation and following checks) are reported in the double entry matrix on (Table 2).

The requested water demands are reported in Table 3. Considering that farmers express preferences only about the day the water is needed, all requests are scheduled to start at the same time.

In this test, all the considered off-takes have the same priorities $(\alpha$ and $\beta$ ). By changing this parameter, it is possible to take into consideration potential priorities and anticipate some irrigations. The available inflow at the district entrance is $320 \mathrm{l} / \mathrm{s}$ that translates to $1152 \mathrm{~m} 3$ per time interval of 60 minutes (see $\mathrm{C}$ in Table 2). This is also assumed as the maximum amount of water available in the network, $r_{i}=1152 \$, i=1, \ldots, N$. 
In this example, every single irrigation request needs much less water than the amount available within the system; however, the total required volume is larger than the one that can be supplied through the network at each time step because of the long duration of many irrigations and the presence of losses due to seepage. For example, the system manager must input to channel 1 almost the double of the water requested on channels 3 and 4 in order to cope with the seepage.

\begin{tabular}{|r|cccc|rrr|}
\hline \multicolumn{1}{|c|}{$\mathrm{k}$} & $\mathrm{i}$ & $\alpha$ & $\beta$ & $\epsilon$ & Hour & $\mathrm{d}(\mathrm{min})$ & $\mathrm{Vm}^{3}$ \\
\hline 1 & 1 & 1 & 1 & 0.8 & 8 & 660 & 72 \\
2 & 2 & 1 & 1 & 0.8 & 8 & 540 & 72 \\
3 & 3 & 1 & 1 & 0.8 & 8 & 360 & 72 \\
4 & 4 & 1 & 1 & 0.8 & 8 & 240 & 72 \\
5 & 1 & 1 & 1 & 0.8 & 8 & 300 & 47 \\
6 & 2 & 1 & 1 & 0.8 & 8 & 360 & 50 \\
7 & 3 & 1 & 1 & 0.8 & 8 & 180 & 90 \\
8 & 4 & 1 & 1 & 0.8 & 8 & 540 & 90 \\
9 & 3 & 1 & 1 & 0.8 & 8 & 360 & 90 \\
10 & 4 & 1 & 1 & 0.8 & 8 & 420 & 54 \\
\hline
\end{tabular}

Tab. 3: Off-take number (k), delivering channel (i), priority coefficients ( $\boldsymbol{\alpha}$ $\boldsymbol{\beta})$, minimum ratio of water that must be delivered $(\boldsymbol{\epsilon})$, duration of the irrigation in minutes (d) and water taken from the channels per time interval (v).

IBM ILOG Cplex 12.9 is the solver used for the final mixed integer linear optimization problem. The considered problem has a total of 2112 continuous and 720 boolean variables, 806 inequality constraints and 620 equality constraints. The network is empty at the beginning of the optimization, with the initial condition in which there is no water in the canals and all the gates are closed.

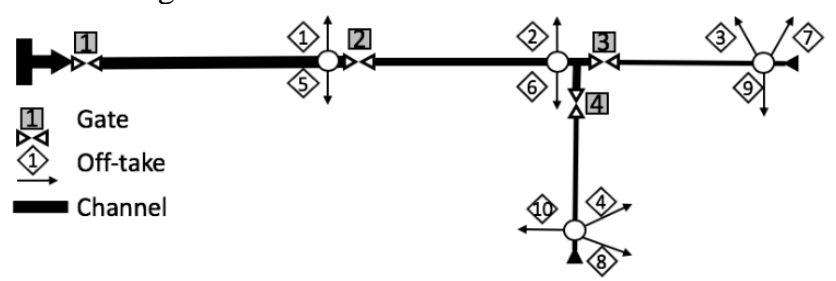

Fig. 1. Schematics of the considered water distribution network. the grey squares represent the gates and the white diamonds the farmers' off-takes.

The optimal scheduling resulting from the run is shown in Figure 2. Because the network has no water at the beginning of the optimization, all irrigations are delayed according to the time needed for the water to go from the district inlet to off-take locations, meaning 2 time intervals for canal 1, 3 for canal 2 and 4 for canals 3 and 4 . The irrigations that do not receive all the requested water are 1,8 and 9 . It is possible to notice that for every canal, the irrigations that begin the earliest are the ones that last the most, while shorter irrigations are delayed.

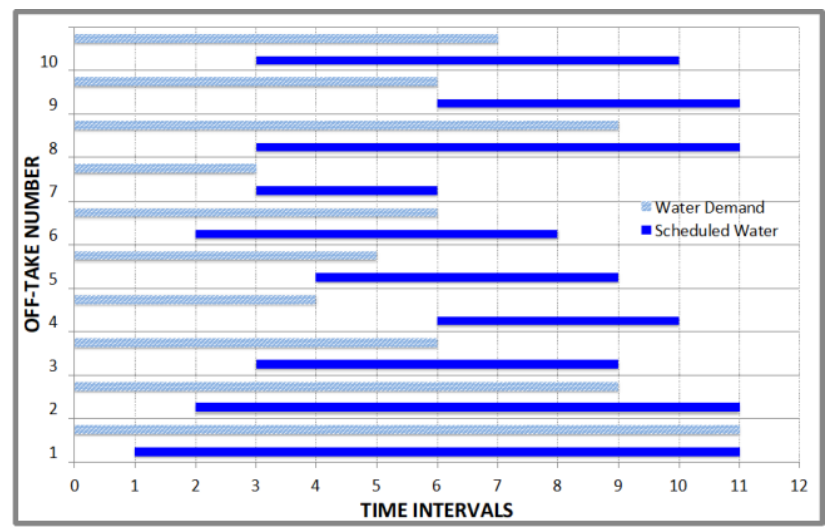

Fig. 2. Demands and scheduled water for the off-takes.

In Table 4 we report the volumes in input and the water stored in every channel for every time interval. It is worth noting that in the time period 4-7, the network is put under intense stress as the maximum respective inlet volumes are almost reached on both channels 1 and 2 . This is due to the large number of irrigations performed in intervals 7 and 8. As a matter of facts, nine out of ten irrigations are active in such time intervals.

\begin{tabular}{|c|c|c|c|c|c|c|}
\hline & 1 & 2 & 3 & 4 & 5 & 6 \\
\hline 1 & $878(0)$ & $878(0)$ & $878(0)$ & $1077(0)$ & $1077(0)$ & $1077(0)$ \\
\hline 2 & $0(0)$ & $630(0)$ & $630(0)$ & $630(0)$ & $743(0)$ & $743(0)$ \\
\hline 3 & $0(0)$ & $0(0)$ & $203(0)$ & $203(0)$ & $203(0)$ & $203(0)$ \\
\hline \multirow[t]{2}{*}{4} & $0(0)$ & $0(0)$ & $180(0)$ & $180(0)$ & $180(0)$ & $270(0)$ \\
\hline & 7 & 8 & 9 & 10 & 11 & 12 \\
\hline 1 & $1077(0)$ & 440 (59) & $440(0)$ & $440(0)$ & $440(0)$ & $440(2)$ \\
\hline 2 & $743(0)$ & $684(0)$ & $280(3)$ & $280(1)$ & $280(0)$ & $350(71)$ \\
\hline 3 & $203(0)$ & $203(0)$ & $203(0)$ & $41(72)$ & $41(0)$ & $41(32)$ \\
\hline 4 & $270(0)$ & $270(0)$ & $270(0)$ & $112(0)$ & $113(0)$ & $113(90)$ \\
\hline
\end{tabular}

Tab. 4: Water volume $\left(\boldsymbol{m}^{3}\right)$ in input to every channel for every time interval of 1 hour. in the brackets the water remaining in the channels in the same time interval.

For what regards the performance of the final scheduling, most of the irrigations receive the requested quantity of water: $95 \%$ of the water requested by the farmers $\left(4689 \mathrm{~m}^{3}\right.$ in total) is delivered to the field. However, these irrigations require the use of $9139 \mathrm{~m}^{3}$ of water within the district, meaning that around $50 \%$ of the water introduced in the system is lost due to seepage or due to canal management. Water losses due to seepage cannot be avoided in case of earthen canals and this application shows how the considered formulation also takes into account this aspect. The water remaining in the canals at the end of the irrigation is $195 \mathrm{~m}^{3}$ (nearly $2 \%$ of the overall volume).

The gatekeeper trajectory is reported in Figure 3. It is possible to notice that the first four operations are performed in order to open the four gates of the network and start the irrigation, while three operations are performed going from gate 1 to gate 4 in order to manage the water during the irrigation. The final three operations are used to close the gates. 


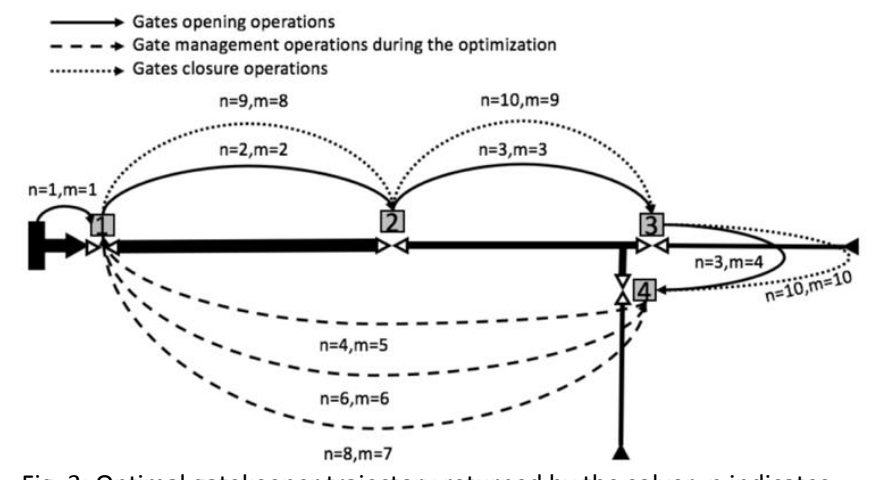

Fig. 3: Optimal gatekeeper trajectory returned by the solver. $\mathbf{n}$ indicates the time interval the operation is performed and $m$ the number of the operation.

The solver performance is summarized in Figure Iref\{fig:synt_gap\}, that shows the values of the gap between the current optimal solution and the lower bound against the computational time. The initial mixed-integer solution has a value of the gap of $98.53 \backslash \%$ that is quickly reduced by the algorithm. Giving an initial feasible solution greatly helps the solver. As a matter of facts, even if the initial integer solution is quite inefficient, Cplex uses methodologies in integer optimization to quickly improve such solution. This explains the fast decrease of the gap in the first half of the optimization. The second part is more focused on improving the lower bound, that is the current continuous solution, so that the gap reaches zero.

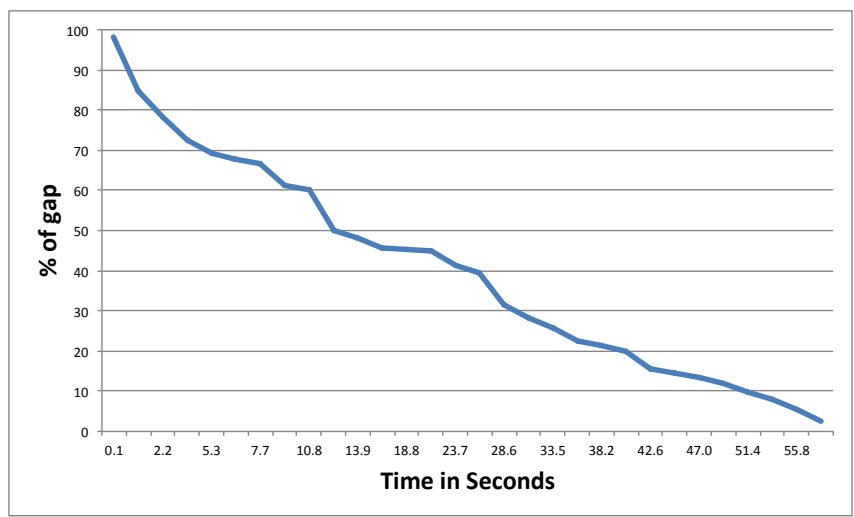

Fig. 4: Gap reduction by the optimization solver.

The results of the optimization model have been verified via hydraulic simulation (Dynamic Wave Analysis) using the SWMM (Storm Water Management Model by EPA Environmental Protection Agency of USA) simulation software [7]. The movement of water through a conveyance network of canals and pipes is governed by the conservation of mass and momentum equations for gradually varied, unsteady free surface flow. The adjustments of the sluice gates have been chosen in a way to ensure, as far as possible, the flow rates predicted by the optimization model, given the difference in physical description of the water passage from one canal to another between the optimization model and the reality-simulating model. Canal 1 is directly powered by a water source fixed at a given level, therefore it is possible to assure perfect correspondence (Figure 4, upper panel). In the following canals, changes in upstream flow regulation, gate openings and irrigations continuously influence the water level, thus making the hydraulic conditions in the river network slightly different from those provided by the optimization model (Figure 4, lower panel).
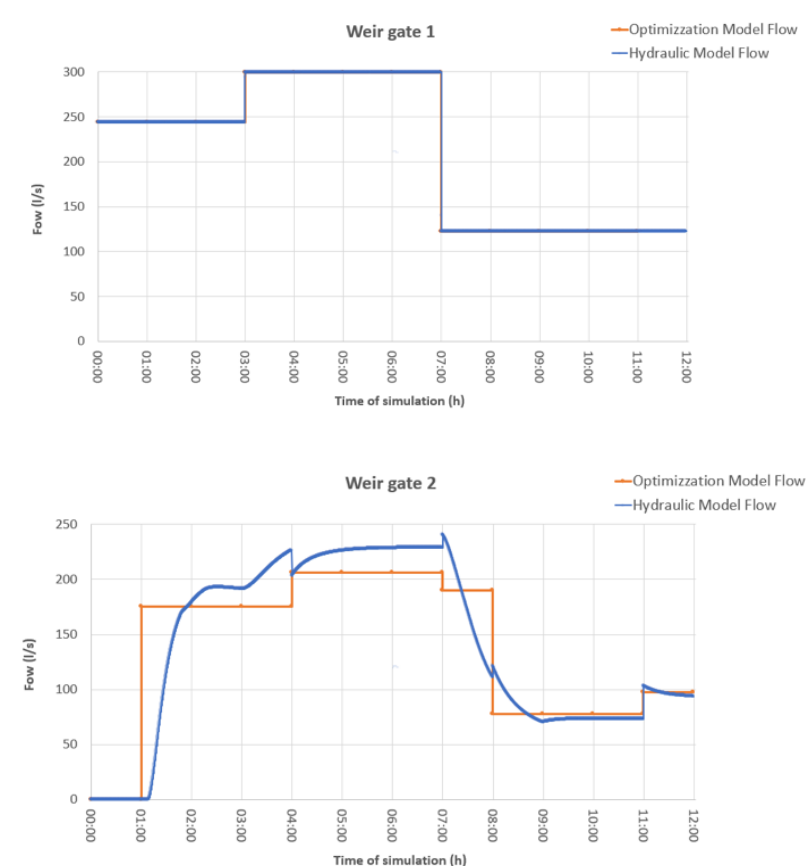

Fig. 4. Comparison of the flow entering into the irrigation district (up) and second canal (down) for the optimization and hydraulic model.

Despite those differences, which were expected in the light of the significant simplifications on reproducing the water dynamic into the optimization scheme, the hydraulic model properly reproduces the overall dynamic of the flowing water and prove that the hydraulic conditions within the canals (i.e. water level, overall volume, retention time, etc.) are adequate to sustain the irrigation. Similar results are observed along all canals. Following the optimal scheduling provided by the optimization tool with the hydraulic model, all irrigations have been satisfied and no critical conditions were observed. These results validate the outcomes of the optimization model that appears reliable in providing a proper irrigation scheduling and operations sequence to WDG called to operate on the network.

\section{CONCLUSIONS}

This work presents a mixed integer linear optimization formulation for the irrigation water scheduling problem that improves traditional solutions by adding the possibility to store water, keeping into consideration the seepage and reducing significantly the number of integer variables. This new formulation is tested on a synthetic benchmark and validated referring to SWMM simulator which allows a close real- representation of the water dynamics. Results validate the outcomes of the optimization model that appears reliable in providing a proper irrigation scheduling and operations sequence to WDG called to operate on the network.

The proposed optimization solution can be easily embedded within the framework of models, repositories and sensors installed in the field. In fact, from this framework can derive the water needs that constitute the input of the optimization problem. Subsequently, the same framework can be used to observe the impact of applying optimized scheduling to test the effectiveness of the approach. 


\section{ACKNOWLEDGMENT}

This research has been jointly funded by the European Commission in Europe and MCTIC/RNP in Brazil, under the EUB-02-2017 IoT Pilots call.

\section{REFERENCES}

[1] Baume J., Sau J. \& Malaterre P. O. (1998). Modelling of irrigation canal dynamics for controller design, SMC'98 Conference Proceedings. 1998 IEEE International Conference on Systems, Man, and Cybernetics, Volume 4, San Diego, pp. 3856-3861.

[2] Ding Y., Wang L., Li Y. \& Li D. (2018). Model predictive control and its application in agriculture: A review. Computers and Electronics in Agriculture, Volume 151, pp. 104-117.
[3] Hashemy Shahdany S. M., Firoozfar A., Maestre J. M., Mallakpour I., Taghvaeian S. and Karimi P. (2018). Operational performance improvements in irrigation canals to overcome groundwater overexploitation, Agricultural Water Management 204, pp. 234-246.

[4] Hong S., Malaterre P. O., Belaud G. \& Dejean C. (2014). Optimization of water distribution for open-canal irrigation networks. Journal of Hydroinformatics, 16(2), 341-353.

[5] Jägermeyr J., Gerten D., Heinke J., Schaphoff S., Kummu M. \& Lucht W. (2015). Water savings potentials of irrigation systems: global simulation of processes and linkages, Hydrol. Earth Syst. Sci., Volume 19, pp. 3073-3091.

[6] Malaterre P.O., Rogers D. \& Schuurmans J. (1998). Classification of Canal Control Algorithms, Journal of irrigation and drainage engineering 124, pp. 3-10.

Rossman, L.A. (2017). SWMM reference manual volume II - hydraulics, US.EPA. 